\title{
Connectivity-based segmentation of retinal vessels in eye fundus images
}

\section{Segmentación basada en la conectividad de la red vascular de la retina en imágenes de fondo de ojo}

\author{
Miquel Ralló, María S. Millán ${ }^{{ }^{*}}$ \\ Grupo de Óptica Aplicada y Procesado de Imagen, Facultat d'Òptica i Optometria de Terrassa, \\ Universitat Politècnica de Catalunya, BARCELONATECH, Terrassa (Barcelona), España \\ ${ }^{(*)}$ E-mail: miquel.rallo@upc.edu, m.millan@upc.edu \\ S: miembro de SEDOPTICA / SEDOPTICA member
}

Received :23/12/2016 Accepted: 18/09/2017

DOI: $10.7149 /$ OPA.50.4.49070

\begin{abstract}
:
A new unsupervised method for segmentation of objects of diverse nature with the common feature of connectivity (e.g. branching trees or net-shaped objects) is proposed. A preferred application to the vasculature segmentation of retinal images has been illustrated using images from DRIVE database. In the pre-processing stage, the method overcomes the common problem of non-uniform illumination of eye fundus images. The method follows with an iterative algorithm that starts with a seed and adds, at each step, a new vessel segment connected to the previously segmented part. The result preserves the connectivity as a distinct feature of the retinal vessel tree. The segmentation performance is evaluated through common signal detection metrics: sensitivity, specificity and accuracy.
\end{abstract}

Key words: Eye fundus image, retinal vasculature, blood vessel segmentation, digital image analysis, computer-aided diagnosis

\section{RESUMEN:}

Se propone un nuevo método no supervisado para la segmentación de objetos de tipología diversa que presenten la conectividad como característica común (por ejemplo, árboles ramificados, redes, etc.). Una aplicación preferente de este trabajo consiste en la segmentación del árbol vascular en imágenes de la retina y ha sido ilustrado mediante el uso de imágenes de la base de datos DRIVE. En la etapa de preprocesado, el método aborda el problema de la iluminación no uniforme, común en las imágenes de fondo de ojo. Seguidamente, utiliza un algoritmo iterativo que parte de una semilla a la que se añaden, en cada bucle, un nuevo fragmento de vaso que se conecta a la parte previamente segmentada. El resultado preserva la conectividad como un rasgo distintivo del árbol vascular de la retina. El rendimiento del método de segmentación se evalúa mediante métricas habituales en la detección de señales: sensibilidad, especificidad y exactitud.

Palabras clave: Imagen de fondo de ojo, sistema vascular de la retina, segmentación de vasos sanguíneos, análisis digital de imágenes, diagnóstico asistido por ordenador.

\section{REFERENCES AND LINKS / REFERENCIAS Y ENLACES}

[1] R. Bock, J. Meier, L. G. Nyúl, J. Hornegger \& G. Michelson, "Glaucoma risk index: automated glaucoma detection from color fundus images," Med Im Anal 14 (3), 471-481 (2010).

[2] R. J. Winder, P. J. Morrow, I. N. McRitchie, J. R. Bailie, \& P. M. Hart, "Algorithms for digital image processing in diabetic retinopathy," Comput Med Imag and Grap 33 (8), 608-622 (2009).

[3] M. Matsui, T. Tashiro, K. Matsumoto \& S. Yamamoto, "A study on automatic and quantitative diagnosis of fundus photographs. I. Detection of contour line of retinal blood vessel images on color fundus photographs (author's transl.)," Nippon Ganka Gakkai Zasshi 77 (8), 907-918 (1973).

[4] S. Chaudhuri, S. Chatterjee, N. Katz, M. Nelson \& M.Goldbaum, "Detection of blood vessels in retinal images using two-dimensional matched filters," IEEE Trans Med Imag 8 (3), 263-269 (1989). 
[5] J. V. Soares, J. J., Leandro, R. M., Cesar, H. F., Jelinek, \& M. J. Cree, "Retinal vessel segmentation using the 2-D Gabor wavelet and supervised classification," IEEE Trans on Med Imag 25 (9), 1214-1222 (2006).

[6] M. M. Fraz, P. Remagnino, A. Hoppe, B. Uyyanonvara, A. R. Rudnicka, C. G. Owen \& S. A. Barman, "Blood vessel segmentation methodologies in retinal images-a survey," Comput Meth Prog Biomed 108, 407-433 (2012).

[7] M. D. Abràmoff, M. Garvin, M. Sonka, "Retinal Imaging and Image Analysis," IEEE Rev in Biomed Eng 3, 169-208 (2010).

[8] B. Al-Diri, A. Hunter, D. Steel, \& M. Habib, "Automated analysis of retinal vascular network connectivity," Comput Med Imag and Graph, 34 (6), 462-470 (2010).

[9] E. M. Sigurðsson, S. Valero, J. A. Atli Benediktsson, J. Chanussot, H. Talbot \& E.Stefánsson, "Automatic retinal vessel extraction based on directional mathematical morphology and fuzzy classification," Patt Recog Lett 47, 164-171(2014).

[10] V. S. Joshi, M. K. Garvin, J. M. Reinhardt \& M. D. Abràmoff, "Identification and reconnection of interrupted vessels in retinal vessel segmentation," Proc 8th IEEE Int Symp Biomed Imag, Nano Macro (ISBI), 1416-1420 (2011).

[11] DRIVE (Digital Retinal Images for Vessel Evaluation), http://www.isi.uu.nl/Research/Databases/DRIVE/

[12] M. Ralló, M. S. Millán, "Connected iterative segmentation of retinal vessels from fundus images", Abstract Book of RIAO-OPTILAS 2016, Moraga, P. and Saavedra, C.; 21-25 November; CEFOP-UdeCConcepción - Chile 2016. Page 222.

[13] STARE (Structured Analysis of the Retina), http://www.ces.clemson.edu/ ahoover/stare/

[14] REVIEW, http://www.aldiri.info/REVIEWDB/REVIEWDB.aspx

[15] E. Trucco et al. "Validating retinal fundus image analysis algorithms: issues and a proposal," Invest Ophthalmol Vis Sci 54, 3546-3559 (2013).

[16] A. Vogt, "Die Ophthalmoskopie im rotfreiem licht," in Graef Saemisch Handbuch der gesamten Augenheilkunde 3, (1925).

[17] J. V. Soares, J. J. Leandro, R. M. Cesar, H. F. Jelinek \& M. J. Cree, "Retinal vessel segmentation using the 2-D Gabor wavelet and supervised classification,” IEEE Trans on Med Imag 25 (9), 1214-1222 (2006).

[18] F. M. Villalobos-Castaldi, Uso de la red vascular de la retina como medio biométrico de identificación, PhD. Tesis, Centro de Investigación en Computación, México D.F., p. 230 (2011).

http://www.repositoriodigital.ipn.mx/handle/123456789/7005

[19] S. Cepeda-Fuentealba, Segmentación de vasos sanguíneos de retina usando selección de características mediantes distancia de bhattacharyya y algoritmos genéticos, para un clasificador por maximización de la entropía, Santiago, Chile: Universidad de Chile - Facultad de Ciencias Físicas y Matemáticas, (2016). http://repositorio.uchile.cl/handle/2250/138129

[20] A. D. Hoover, V. Kouznetsova \& M. Goldbaum, "Locating blood vessels in retinal images by piecewise threshold probing of a matched filter response," IEEE Trans on Med Imag 19 (3), 203-210 (2000).

\section{Introduction}

Segmentation of blood vessels from retinal fundus images is a major issue in retinal image analysis. Sometimes, the vessel tree is the structure of main interest, but in some other cases, this tree is an obstacle to remove prior to study the background and occluded fundus area (see, for instance, Ref. 1 for glaucoma detection and Ref. 2 in the field of diabetic retinopathy). Blood vessels, more specifically vessel centrelines and bifurcations, have been used as landmarks in image registration or spatial alignment of images, which is essential in longitudinal monitoring of the retinal appearance. Retinal vasculature is a valuable indicator for a variety of diagnoses including diabetes, hypertension and arteriosclerosis. It is commonly agreed that retinal vessel segmentation is a basic step in the development of most image-based diagnostic methods. In fact, the first method published for retinal image analysis [3] primarily focused on vessel segmentation. Since the early work of S. Chaudhuri et al. [4], who performed a segmentation of retinal vessels by matched filters, image analysis researchers continue developing this subject with 
hundreds of increasingly innovative works $[5,6]$. Their approaches are usually classified into two main categories: supervised and unsupervised methods.

Supervised methods classify pixels into vessel and non-vessel categories, based on a previously known classification of similar pixels into those two categories. The classification method runs over a set of vectors, whose components are selected features associated to each pixel describing the pixel itself and its neighbouring area. Unsupervised methods do not require any training set and encompass a wide range of image processing techniques: morphological methods, matched filters, wavelet analysis, etc. A deeper insight into the methods corresponding to those categories and their development can be found in the excellent reviews by Abràmoff et al. [7] and from Fraz et al. [6]. Despite the progress already accomplished, there are still challenges in retinal image segmentation and an active research can be acknowledged on smaller vessel detection, separation of arteries and veins, vessel segmentation in images with substantial pathology, assessing accurate vessel diameter; vessel tortuosity, and vessel tree analysis including tree branching patterns [7]. Vascular connectivity, however, appears to be addressed only in relatively little published work [8-10]. One of them is the algorithm proposed by Al-Diri et al. [8] that forms a retinal vessel graph by analysing the potential connectivity of segmented retinal vessels. Selforganizing feature maps are used to model implicit cost functions for the junction geometry and resolve the configuration of local sets of segment ends, thus determining the network connectivity. The algorithm was tested on junctions drawn from the public domain DRIVE database [11]. Sigurðsson et al. [9] had already considered the so-called linear connectivity of vessels to segment them. Their method is based on directional mathematical morphology, namely path opening to detect filiform structures, and fuzzy classification. In Sigurðsson's approach the role of connectivity is applied at a local level and the method does not ensure a connected global result. Joshi et al. [10] developed a method to reconnect vessel segments identified by a neural network classifier. The set of the long enough connected components are subsequently classified into primary vessel network and interrupted vessel segments. The latter are then connected to the primary network. But the final reconnected vessel network can be still a disconnected set of vessels because it is not assured that the primary network is fully connected.

In this paper we propose a new method, belonging to the class of unsupervised methods, that takes advantage from the connected nature of the vascular tree of the retina [12]. It basically consists in an iterative method that adds, at each step, a new vessel segment connected to the previously segmented part. The result preserves the connectivity as a distinct feature of the retinal vessel tree captured in eye fundus images. In case of two o more disjoint vessel trees existing in a given image, a repeated application of the method would allow the separation of each individual tree from the rest.

The proposed method consists of a preprocessing stage followed by an iterative region growing and segmentation process. The preprocessing stage prepares the region of interest and enhances the retinal vasculature. The iterative process starts with the segmentation of an initial seed, being a connected segment of the retinal vasculature, to which new segments join in subsequent iterations on the basis of connectivity. The method is intended to solve the segmentation of an unevenly illuminated object from an uneven background, just by assuming that the object is topologically connected. Apart from the assumption of topological connectivity, which plays an essential role in the iteration procedure, our method does not require any other specific feature from the image under analysis except for the vessel scale: the approximate width in pixels of the largest vessels is necessary for a proper segmentation of the object seed from the background. For these reasons, although we illustrate the applicability of the proposed segmentation method to the retinal vasculature, it could be also applied to the segmentation of objects of diverse nature with the common feature of connectivity (e.g. branching trees or net-shaped objects).

In order to compare our results to those derived from other known segmentation methods we have used DRIVE (Digital Retinal Images for Vessel Evaluation) database. This database was established to enable comparative studies on segmentation of retinal blood vessels in retinal fundus images. It contains 40 fundus images from subjects with diabetes, both with and without retinopathy, as well as retinal vessel segmentations manually performed by two human observers. Researchers have been invited to test their algorithms on this database and share their results with other researchers through the DRIVE website. A few datasets (DRIVE, STARE [13] and REVIEW [14]) provide an easily accessible reference standard and that is why they are widely used for validating automatic retinal image analysis algorithms that deal with retinal vasculature. However, they suffer from some limitations too [15]: a lack of objective definition of the location of the edge of a retinal blood vessel, a labour-intensive annotation task for vessel mask 
generation, a difficult vessel versus non-vessel classification of pixels located at vessel edges, and a variety of standards with segmentation provided by human observers to assess detailed as well as overall algorithm performance.

In this paper, the performance of the proposed segmentation algorithm is evaluated within the fundus image regions defined by the DRIVE database through accuracy, sensitivity and specificity. The results are compared with other results provided in the related literature.

\section{Image analysis method}

The image analysis method is schematically represented in Fig. 1 and described in this section. The sequence of steps is fully illustrated taking the test image \#1 of DRIVE database as an example (Fig.2a).

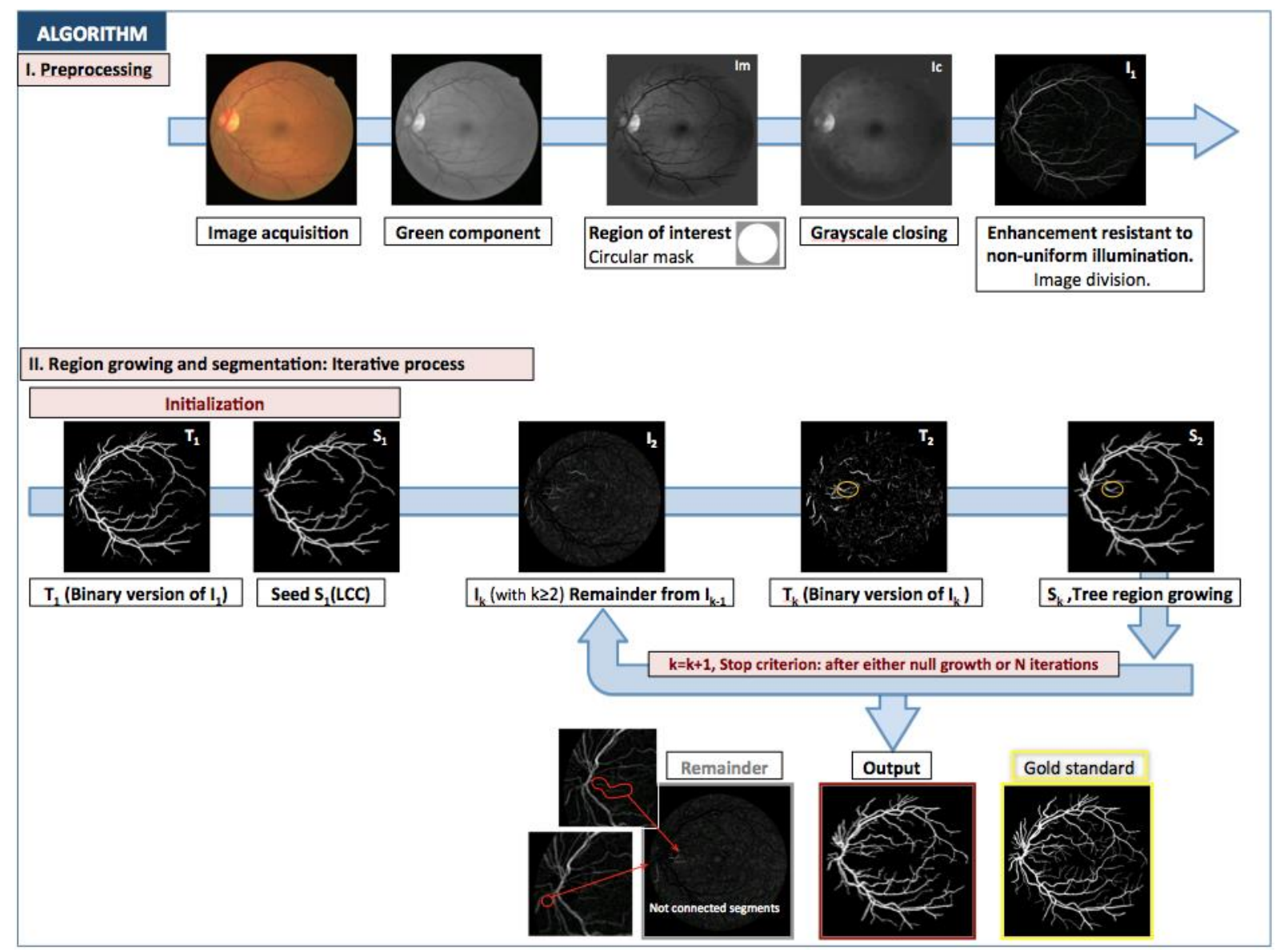

Figure 1. Block diagram of the proposed method

\section{2.a. Preprocessing}

The acquired fundus image is typically a digital colour image (Fig. 1, top part: I. Preprocessing and Fig. 2a). However, for vessel segmentation purposes it is reliable to work on the green channel of the RGB colour space because it exhibits better vessel to background contrast than the other red and blue channels. This practice originates in 1925, when Vogt [16] described the use of green light to enhance the visual contrast of anatomical details of the fundus and coined the term "red-free". The technique is still used today in fundus photography [15]. The extraction of the G (green) component from RGB colour images is also common practice in digital image analysis of the retinal vasculature and has been highly reported (see, for example Refs. 1, 2, 17).

Once the G component has been extracted, we proceed to apply a mask to separate the black corners from the circular-shaped region of interest (ROI) that contains the eye fundus information. We will extend then an average background to cover till the corners of the image by replacing the black pixels with grey pixels. Although the DRIVE database provides a mask for its own image samples, we consider a more general case and generate a mask for the fundus image under study. From the intensity values, pixels are classified 
into two categories (dark and bright) according to the outcome of the K-means method applied to the green channel. Initially, the mask is the complementary of the smallest disk containing all bright pixels. To reduce the border effects, we further erode the circular edge of the ROI with a structural element (square) of $n \times n$ pixels before replacing the masked pixels with grey values. For DRIVE images, the size of the structural element was manually fixed to $n=7$ pixels. All the masked pixels (Fig. 2b) are in-painted and set to the median value of the pixels within the ROI. The median is preferred to the mean value because it is more robust to the relatively few extreme values of the optic disc (oversaturated) and the vessels (under saturated). At this stage, pixels of the ROI remained unchanged (Im, Fig. 2c).

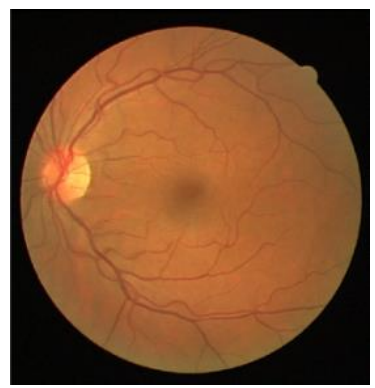

(a)

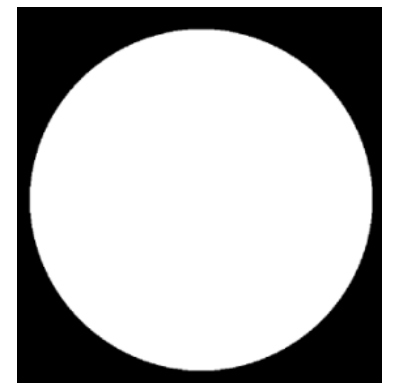

(b)

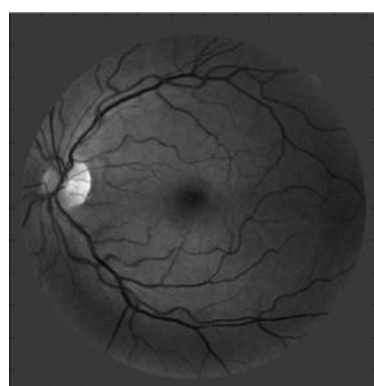

(c)

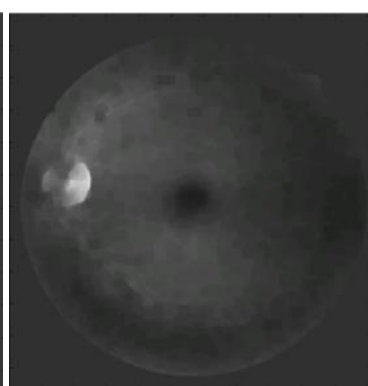

(d)

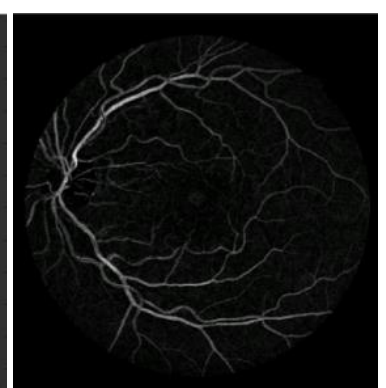

(e)

Figure 2. (a) Test image \#1 from DRIVE database; (b) mask; (c) Im, green component with masked pixels set to the median of the circular ROI pixel values; (d) $I c$, closing of $\operatorname{Im}(\mathrm{c})$; and (e) $I_{1}=(I c / I m)$ : pixel-to-pixel division of (d) and (c). For display purposes exclusively, $\mathrm{I}_{1}$ is converted into grayscale image with range [0...255].

In the last step of the preprocessing stage, we aim to enhance the retinal vasculature within the ROI despite its non-uniform illumination. To do this, we locally estimate the background intensity along the vessels by applying a morphological closing operator to the image Im (Fig. 2c) with a disk-shaped structuring element of radius large enough to cover the largest vessels. In the example that illustrates the method, the largest vessels were 13 pixels width, so the radius of the structuring element was set to 7 pixels. The resulting image $(I c)$ is shown in Fig. $2 \mathrm{~d}$. As intended, the closing operator removes image details with size smaller than the structuring element, specifically blood vessels, thus resulting in an image Ic with local background estimation. The vascular tree can be now enhanced despite non-uniform illumination by dividing the last two grayscale images. The resulting image $\left(I_{1}\right)$ is then obtained by calculating $I_{1}=I c / I m$ on a pixel-to-pixel basis (Fig. 2e). To avoid singularities, if $\operatorname{Im}(p, q)=0$, then $I_{1}(p, q)=\max \left(I_{1}(x, y)\right)$ with $\operatorname{Im}(x, y) \neq 0$. Note that $\min \left(I_{1}(x, y)\right) \geq 1.0$. The background pixels show values close to one in $I_{1}$ because their intensities in $I c$ and $I m$ were similar. Therefore, background pixels appear black in the grayscale image $\mathrm{I}_{1}$ shown in Fig. 2e. But the values of the pixels corresponding to vasculature are much higher because their denominators in Im were lower (vessels appear dark in $I m$ ). Consequently, vasculature pixels appear bright in $I_{1}$. As a result of the preprocessing part, image $I_{1}$ provides an enhanced version of the retinal vasculature on a dark background (Fig. 2e).

\section{2.b. Iterative algorithm for region growing and segmentation}

Although the vasculature appears enhanced throughout image $I_{1}$ (Fig. 2e), only a coarse segmentation would be obtained by binarising with a simple threshold operator. In fact, this rough estimation can be used to obtain a seed for the iterative process, as we will describe later.

Due to the combined effects of the 3D nature of vessels, noise, and lack of proper illumination, three problems, at least, may arise in analysing the pixel values of image $I_{1}$ : 
- Some background pixels can have an intensity value even higher than some object pixels at a local level. See, for instance, Fig. 3a. We will try to overcome this problem using basically the connectivity criterion.

- Some object pixels have too low intensities and are misclassified as background or produce a gap between object segments, Fig. 3b.

- Some vessels may emerge separated or non-connected to the main vessel tree, particularly in the optic disc vicinity. In such a case, since there are no pixels connected to the tree, the algorithm will keep such vessels apart. This fact can be used precisely to isolate the non-connected vessels from the main tree, Figs. 3b and 3c.

We propose an iterative algorithm for region growing and vessel tree segmentation. A large initial seed can be obtained by applying a threshold to image $I_{1}$ and selecting the largest connected component. The relative area of the vasculature to the whole ROI is roughly around 10\% (the percentage of pixels manually labeled as vessels in the ROI of some DRIVE images has been calculated and reaches $10 \%$ in [18], $12,7 \%$ and $12,3 \%$ on average for the training and test DRIVE groups in [19], while Hoover reported about $8 \%$ to $11 \%$ using their 20 fundus images [20]). We consider a threshold $t_{1}$ set to the $87.5^{\text {th }}$ percentile value of intensity within the ROI of $I_{1}$, so that $12.5 \%$ pixels of the ROI have their intensities above the threshold and $87.5 \%$ below. Let $T_{1}$ be a binarised version of $I_{1}$ with threshold $t_{1}, T_{1}=I_{1} \geq t_{1}$. With this threshold we assure that most vasculature pixels have their values higher than $t_{1}$ and will appear in white in the binary image $T_{1}$ (Fig. 4a). Pixels of $T_{1}$ are binary and only take two possible values $\{0,1\}$ that are displayed as \{black, white $\}$. Image $S_{1}$ starts with the initial seed, which is defined as the largest connected component (LCC) found in the binary image $T_{1}$ (Fig. 4b)). As the iterative algorithm runs, the seed placed in $S_{1}$ is going to grow.

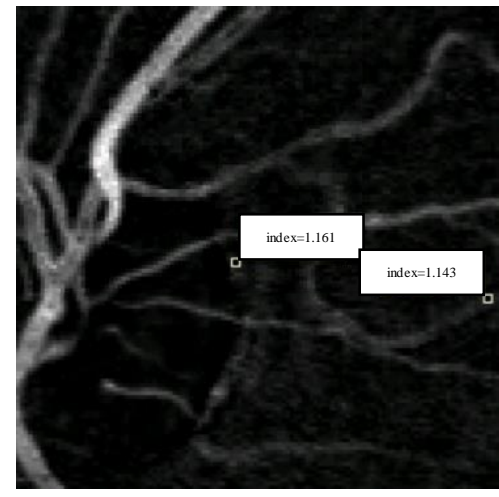

(a)

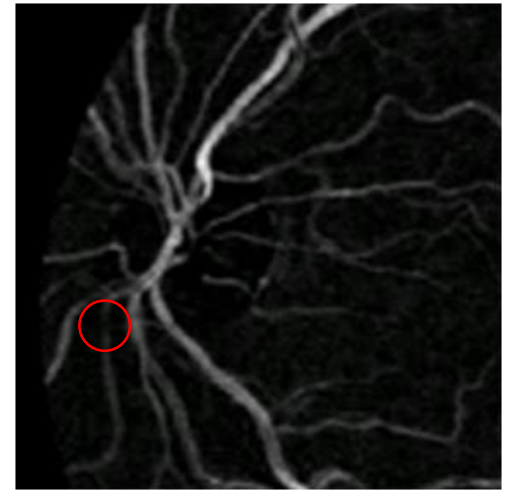

(b)

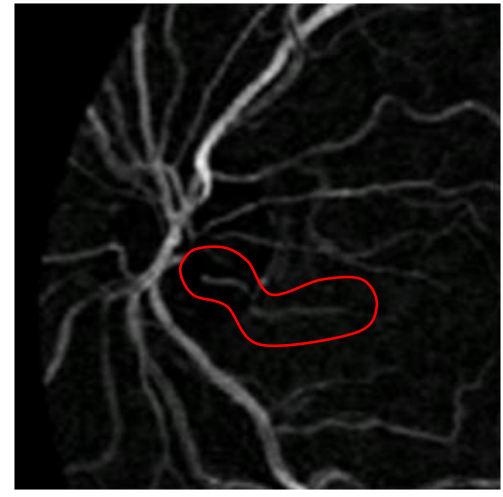

(c)

Figure 3 (a) A background pixel shows higher value than a vascular pixel, (b) Gap caused by vessel pixels with too low values and (c) Vessel emerging from the optic disc that is not connected to the main vasculature structure.

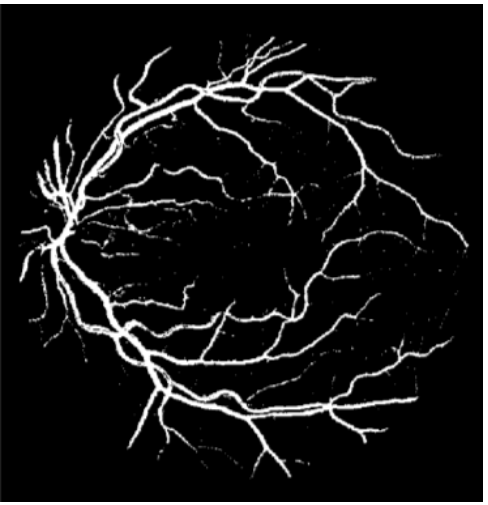

(a)

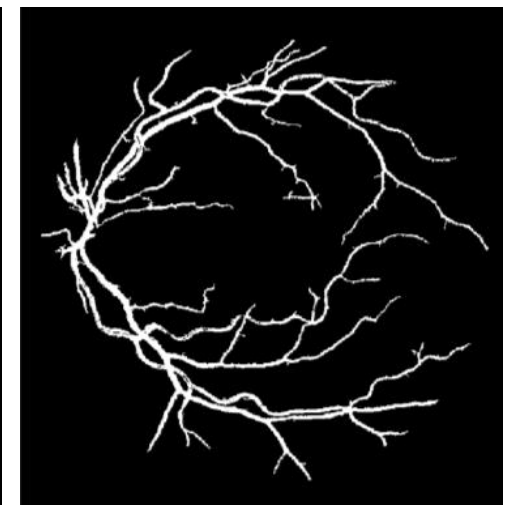

(b)

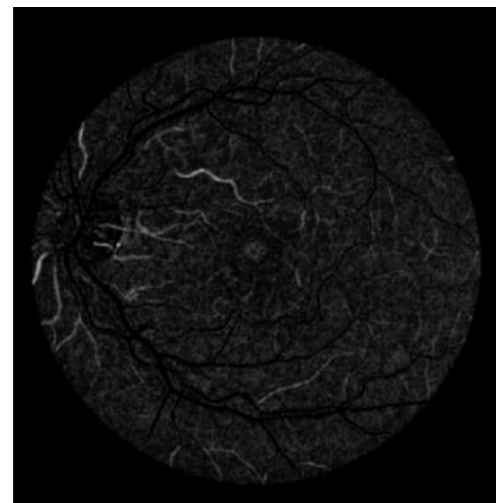

(c)

Figure 4. (a) Image $T_{1}: I_{1}$ thresholded at the $87.5^{\text {th }}$ percentile of the ROI, (b) $S_{1}$ : largest connected component (LCC) of $T_{1}$ and (c) Remainder, $I_{2}$, obtained from $I_{1}$ after removing $S_{1}$.

The remainder $I_{2}$ is built from image $I_{1}$ by removing the pixels belonging to the LCC and setting their values equal to one (Fig. 4c). We recall that the lowest value in image $I_{1}$ is 1 and corresponds to the 
background pixels. Therefore, the remainder $I_{2}$ is greyscale and contains all vessel segments that should be added to $S_{1}$ in the following iterations to obtain the whole vessel tree.

Each iteration starts with $I_{k}, k \geq 2$, which is the greyscale remainder from which we have already removed part of the vasculature. The vasculature currently segmented is contained in the binary image $S_{k-1}$. We ignore the amount of vessel pixels remaining in $I_{k}$, but we certainly know that they must be among the brightest. In this point, we apply a restrictive threshold that selects just those pixels within the $5 \%$ top high values of $I_{k}$. In other words, the threshold $t_{k}$ is set to the $95^{\text {th }}$ percentile value of intensity within the ROI of $I_{k}$. The result is the binary image $T_{k}=I_{k} \geq t_{k}\left(T_{2}\right.$ is shown in Fig. 5a). $T_{k}$ usually contains a non-connected set of vessel segments along with some noisy pixels, the latter likely belonging to the background. There is no certainty that some element in $T_{k}$ may be in connectivity with the currently segmented object in $S_{k-1}$. Therefore we dilate $S_{k-1}$ until it overlaps $T_{k}$. We consider the set of pixels $S_{k-1}^{\text {dil }}$ whose distance to $S_{k-1}$ is smaller than or equal to $d_{k}=\operatorname{dist}\left(S_{k-1}, T_{k}\right)$. Note that $S_{k-1}^{\text {dil }}$ contains $S_{k-1}$ and shares some pixel with $T_{k}$. So there is, at least, one element of $T_{k}$ in connectivity with $S_{k-1}^{d i l}$. There might be more than one segment in $T_{k}$ contacting $S_{k-1}^{d i l}$, but we consider just one segment -the largest one- to join per iteration, as we show next. Recall that $S_{k-1}^{d i l}$ and $T_{k}$ are binary images, so $\max \left(S_{k-1}^{d i l}, T_{k}\right)$ is a binary image too. Now, in this last binary image, we take the connected component $C_{k}$. This component $C_{k}$ contains $S_{k-1}$ and enlarges it with new segments. In order to identify which segments of $T_{k}$ should be added to $C_{k}$ at this step, we consider $\min \left(C_{k}, T_{k}\right)$, the part of $C_{k}$ originating in $T_{k}$. We finally pick the largest connected component $L C C_{k}$ among the former set of segments.

The distance from $S_{k-1}$ to $L C C_{k}$ is equal to $d_{k}$. If $d_{k}>1$, we still need to connect $L C C_{k}$ to $S_{k-1}$. We take $S_{k}$ as the set of pixels containing $S_{k-1}, L C C_{k}$, and the pixels whose distances to $S_{k-1}$ and $L C C_{k}$ add up by no more than $d_{k}$.

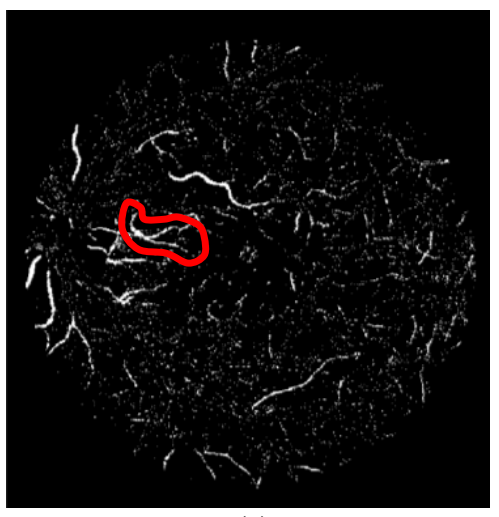

(a)

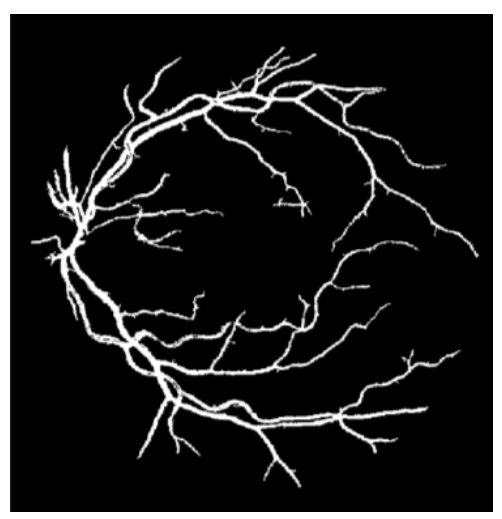

(b)

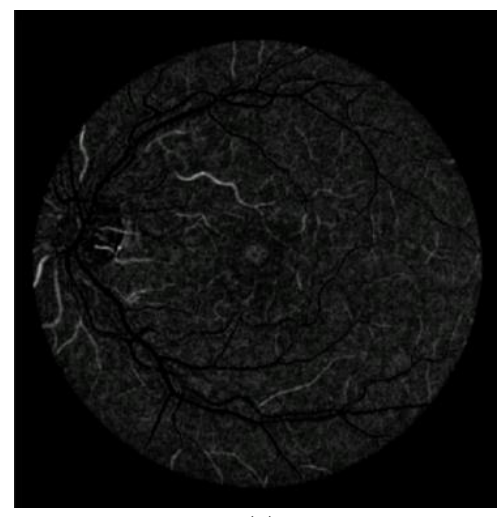

(c)

Figure 5. (a) Image $T_{2}: I_{2}$ thresholded at the $95^{\text {th }}$ percentile of the ROI, showing the $\mathrm{LCC}_{2}$ of $T_{2}$ among those closest to $S_{1}$, (b) $S_{2}: S_{1}$ enlarged with this connected component and (c) Remainder $I_{3}$ obtained after removing $S_{2}$ from $I_{2}$.

The last step of the iteration consists in obtaining the remainder grayscale image $I_{k+1}$ (Fig. 5c) from image $I_{k}$ by setting to one the intensities of the object pixels contained in $S_{k}$, which is in turn the image where the segmented object (vessel tree) is growing. A new iteration is then performed until the stopping criterion is reached.

The iterative loop breaks when there is no growth of the segmented object, i. e. when $S_{k}=S_{k-1}$. Otherwise, the segmentation algorithm finishes after 500 iterations. That maximum number of iterations has been manually established for the specific analysis of DRIVE database images and has been experimentally derived from the training images, as it will be shown in the next section.

\section{Results and discussion}

By applying the proposed method to the test image \#1 (Fig. 2a), we have obtained the result shown in Fig. 6a. DRIVE database provides manual segmentations of the vasculature done by human observers, which can be used as ground truth (Fig. 6b) for comparison with the results obtained with of our method. Finally, Fig. $6 \mathrm{c}$ shows the final remainder when the iterative algorithm stopped. 


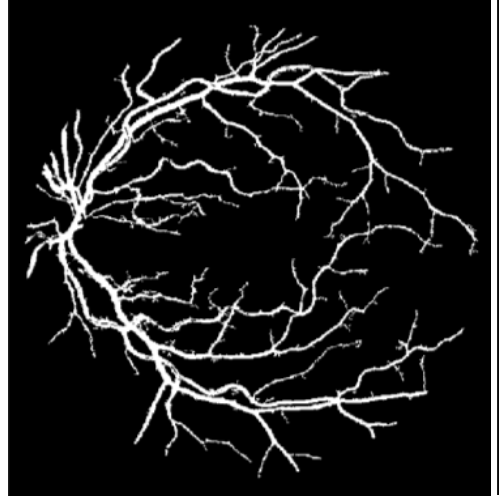

(a)

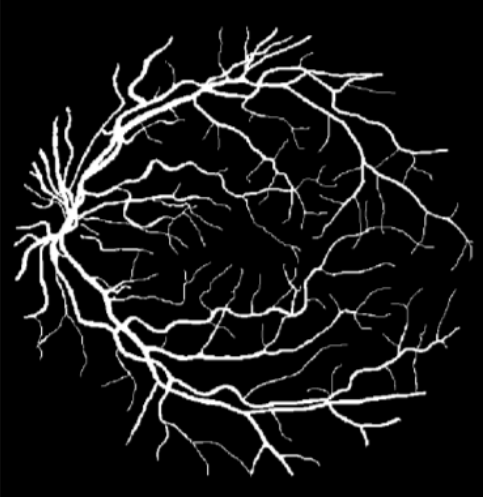

(b)

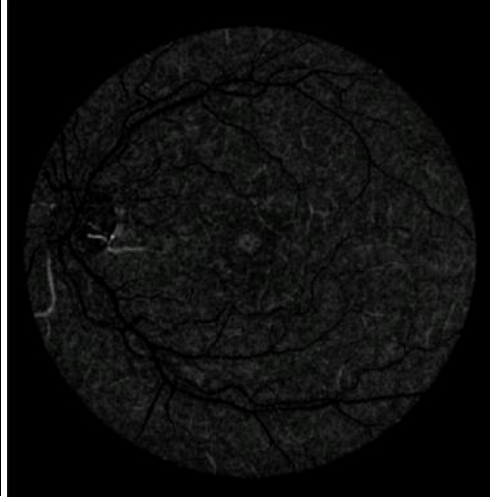

(c)

Figure 6. (a) Connected vascular tree segmented by the proposed method, (b) ground truth provided by DRIVE database, and (c) remaining segments of the vasculature non-connected to (a).

To evaluate the performance of the proposed method we compute some common signal detection metrics: sensitivity (pixels corresponding to retinal vessels correctly identified), specificity (pixels not corresponding to retinal vessels correctly identified), and accuracy (pixels correctly identified). For the sake of comparison, we apply the mask provided by DRIVE database to our result prior to calculation. Table 1 shows the variation of such metrics versus the number of iterations for the test image \#1. Whereas the sensitivity improves with the iteration number, the specificity and accuracy worsen. More specifically, from 50 iterations to 500 iterations the sensitivity improves nearly 4.5\%, whereas the accuracy only decreases a $0.34 \%$. From 500 to 1000 iterations, however, the sensitivity improvement slows down to $1.5 \%$, whereas the accuracy falls almost $0.4 \%$. For this reason, we establish the number of iterations to 500 .

\begin{tabular}{|c|c|c|c|}
\hline Iterations & Sensitivity & Specificity & Accuracy \\
\hline 50 & 0.7281 & 0.9792 & 0.9464 \\
\hline 100 & 0.7364 & 0.9775 & 0.9460 \\
\hline 250 & 0.7593 & 0.9726 & 0.9447 \\
\hline 500 & 0.7726 & 0.9686 & 0.9430 \\
\hline 1000 & 0.7876 & 0.9618 & 0.9391 \\
\hline
\end{tabular}

Table 2. Values of sensitivity, specificity and accuracy obtained with the proposed method (500 iterations)

\begin{tabular}{cccccccccc}
\multicolumn{10}{c}{ for all the 20 test images of DRIVE database. } \\
\hline Image \# & $\mathbf{2}$ & $\mathbf{3}$ & $\mathbf{4}$ & $\mathbf{5}$ & $\mathbf{6}$ & $\mathbf{7}$ & $\mathbf{8}$ & $\mathbf{9}$ & $\mathbf{1 0}$ \\
\hline Sensitivity & 0.7365 & 0.6636 & 0.6816 & 0.6751 & 0.6183 & 0.6407 & 0.5633 & 0.6482 & 0.6762 \\
Specificity & 0.9725 & 0.9777 & 0.9681 & 0.9794 & 0.9791 & 0.9696 & 0.9792 & 0.9799 & 0.9800 \\
Accuracy & 0.9374 & 0.9320 & 0.9299 & 0.9381 & 0.9282 & 0.9261 & 0.9271 & 0.9409 & 0.9437 \\
\hline \multicolumn{1}{c}{} & & & & & & & & \\
\hline 11 & $\mathbf{1 2}$ & $\mathbf{1 3}$ & $\mathbf{1 4}$ & $\mathbf{1 5}$ & $\mathbf{1 6}$ & $\mathbf{1 7}$ & $\mathbf{1 8}$ & $\mathbf{1 9}$ & $\mathbf{2 0}$ \\
\hline 0.7097 & 0.6649 & 0.6819 & 0.7411 & 0.6690 & 0.7050 & 0.6693 & 0.7601 & 0.8186 & 0.7471 \\
0.9703 & 0.9737 & 0.9672 & 0.9718 & 0.9669 & 0.9748 & 0.9723 & 0.9657 & 0.9734 & 0.9727 \\
0.9366 & 0.9350 & 0.9268 & 0.9447 & 0.9359 & 0.9395 & 0.9350 & 0.9421 & 0.9548 & 0.9487
\end{tabular}

We have applied the method to the 20 test images of the DRIVE database, keeping the maximum number of iterations equal to 500. The results have been assessed in terms of the signal detection metrics as shown in Table 2. The mean values of sensitivity, specificity and accuracy are given in Table 3 and compared with the mean values computed from a set of 35 different retinal vessel segmentation methods reported in [6]. Fig. 7 further represents this comparison by placing our scores (dots) among those reported by Fraz et al. (box-plots with its median values represented by vertical segments).

Our method shows better specificity than and similar accuracy to the median of the methods considered by Fraz et al. [6], but lower sensitivity. This can be explained in part by the constraint of connectivity we 
have considered in the initial assumptions. In comparison with the manual labeling of vessels carried out by human observers (Fig.6b), our method is not able to overcome some relatively big gaps such as, for instance, those shown in Fig.3b and 3c, while the subjective interpretation of observers is. This fact affects the sensitivity.

But there might be some cases in which the structure of the object must be taken into account before calculating a meaningful sensitivity. Let us assume, for example, that the vasculature tree has not the appearance of a single tree, but of two non-connected trees, one of them branching to the superior part of the fundus and the other branching to the inferior part of the fundus. This case is not a rare; on the contrary, it typically appears when the field of view does no contain the optic disc where the vessels naturally converge. In such a case, the proposed method, which is essentially based on connectivity, would segment only one of the vessel trees. In a semi-automated application of the proposed method, i.e. with the intervention of a "human in the loop" [15], the iterative process could be restarted to the remainder image to segment the second vessel tree. In this way, the proposed method is useful to separately segment the individual trees.

Table 3. Mean values of sensitivity, specificity and accuracy computed for the segmentation methods applied to DRIVE database that are reported in [6].

\begin{tabular}{lccc}
\hline \multicolumn{1}{c}{ Mean values } & Sensitivity & Specificity & Accuracy \\
\hline Methods reported in Ref. 6 & 0.7271 & 0.96857 & 0.93583 \\
Proposed Method & 0.692 & 0.973 & 0.937 \\
\hline
\end{tabular}

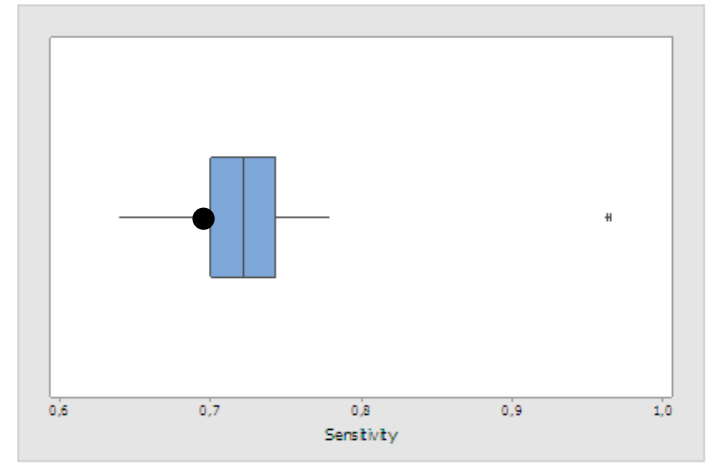

(a)

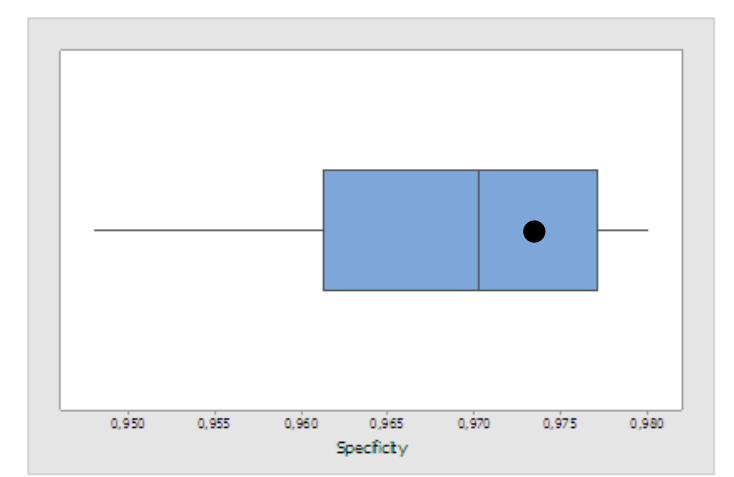

(b)

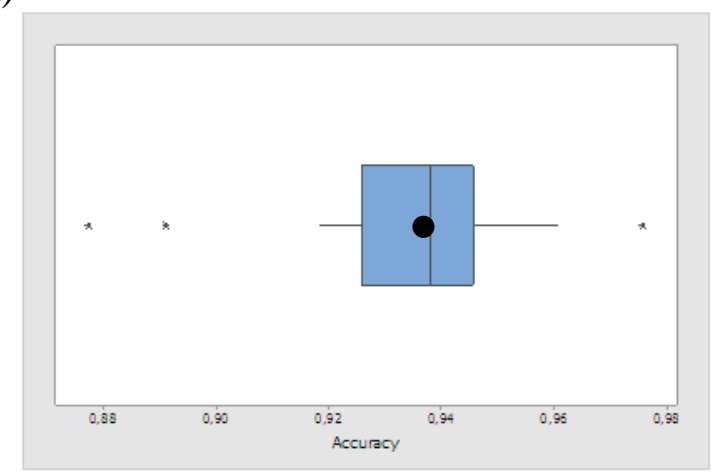

(c)

Figure 7. Box-plots: Mean values of sensitivity (a), specificity (b), and accuracy (c), computed for the 35 segmentation methods applied to DRIVE database that are reported in [6]. The vertical segment inside each box-plot indicates the median value. Dots: values obtained with the proposed method.

\section{Conclusions}

We have proposed an unsupervised method for segmentation of objects of diverse nature with the common feature of connectivity (e.g. branching trees or net-shaped objects). A preferred application to the vasculature segmentation of retinal images has been illustrated using images from DRIVE database. The method takes advantage from the connected nature of the vascular tree of the retina and uses an iterative algorithm that starts with a seed and adds, at each step, a new vessel segment connected to the 
previously segmented part. The result preserves the connectivity as a distinct feature of the retinal vessel tree. In case of two o more disjoint vessel trees existing in a given image, a repeated semi-automatic application of the method would allow the separation of each individual tree from the rest.

Our algorithm has an overall performance similar to other 35 methods reported in [6] when they are applied to DRIVE database images: similar accuracy and slightly better specificity. The sensitivity is somewhat lower because only branches connected to the tree are eventually joined in the iterative process.

The algorithm allows us to separate connected tree-shaped objects from other individual non-connected branches or elements. In addition to eye fundus images, the algorithm can be used to segment tree-shaped objects in a variety of images (nets, nerves, fibers,...).

\section{Acknowledgements}

This research work has been supported by projects DPI2013-43220-R and DPI2016-76019-R, with funds from the Spanish Ministerio de Economía y Competitividad and FEDER. 